\title{
Wandel der Kulturlandschaft auf Stromboli
}

Auf den äolischen Inseln vollzieht sich, ähnlich wie in anderen bis vor kurzem unveränderten Landschaften des Mittelmeerraumes, ein sichtbarer Kulturlandschaftswandel. Dieser wird hauptsächlich durch zwei Faktoren bedingt: den seit dem 2. Weltkrieg aufkommenden Tourismus einerseits und die Entvölkerung anderseits, dieselben also, die wir z. B. auch in bestimmten Bereichen der Alpen kennen. MIKUS ${ }^{1)}$ hat über die wirtschaftsgeographischen Probleme der Inselgruppe publiziert. Wir möchten im folgenden auf eine weniger bekannte Arbeit von FAMULARO ${ }^{2)}$ Professor für Geographie am Istituto Tecnico Commerciale Rom, hinweisen.

Die Arbeit famularos handelt vom ländlichen Haus auf Stromboli, welches letztere er in seiner Abhängigkeit von Natur und Wirtschaft untersucht. Er stellt fest (1971), daß sich das Haus auf Stromboli trotz zahlreicher Erneuerungstendenzen nach wie vor «dem Vulkan unterordnet», und sich damit von demjenigen auf anderen äolischen Inseln unterscheidet. Die Ausführungen FAMULAROS erscheinen uns deshalb besonders wertvoll, weil die Entwicklung auf Stromboli noch nicht so stürmisch verlief wie z.B. auf Lipari oder Vulcano. Die beiden eingangs erwähnten Strömungen, Tourismus und Entvölkerung, haben zur Folge, daß verlassene Wohnplätze sukzessive neu genutzt werden.

Warum sind die Bewohner der Insel Stromboli weitgehend von der traditionellen Wirtschaftsweise abgekommen? Trotz fruchtbarer Vulkanböden behinderten schon früh Wassermangel und Bodenzersplitterung die Landnutzung. So wurde bis zum ersten Weltkrieg, als die Einwohnerzahl ihren Höchststand erreichte, mit den Segelschiffen der Inselbewohner Getreide aus Taranto importiert. Noch heute erkennt man in der Ortschaft Stromboli die Ruinen der dampfbetriebenen Mühle mit einem markanten viereckigen Backsteinkamin. Dem Rückgang der Landwirtschaft steht, vor allem durch den sommerlichen Tourismus bedingt, eine steigende Nachfrage nach Obst, Gemüse, Wein usw. gegenüber.

Fragen wir nach den Gründen für den touristischen Aufschwung der Insel, so sind zu nennen: verbesserte Verkehrsverbindungen: rasche Tragflügelboote, Direktverbindung mit Neapel. Anderseits die Attraktivität der Landschaft: tätiger Vulkan, sauberes Wasser, schwarzer Sandstrand. Ambiente der Siedlung: keine Autos, keine öffentliche Stromversorgung usw. Der überwiegende Teil der Feriengäste stammt aus industrialisierten oder städtischen Gebieten Italiens. Um das attraktive Image Strombolis zu erhalten, müßte wohl weiterhin eine behutsame "Entwicklung» angestrebt werden. Eine Voraussetzung dafür wäre allerdings ein gewisses Verständnis für das Wesen und Werden der Kulturlandschaft, zu welchem die Arbeit von FAMULARO in bester Weise beiträgt. Im folgenden möchten wir auf einige Aspekte derselben eingehen. Auf Stromboli können drei Haustypen unterschieden werden:

1. Wohnteil über dem Wirtschaftsteil

2. Wohnteil neben dem Wirtschaftsteil

3. Wohnteil und Wirtschaftsteil getrennt

Aus den ursprünglich einräumigen («monocellulare») Gebäuden wurden im Laufe der Zeit zwei-, drei- und vierräumige, wobei diese meist in einer Zeile, gelegentlich zwei und zwei übereinander angeordnet sind. Die allermeisten Räume weisen einen nahezu quadratischen Grundriß mit einer Seitenlänge von 5,0-5,4 Metern auf. Das Baumaterial lieferte weitgehend der Vulkan. Bemerkenswert die Dicke der Mauern: 60 Zentimeter und mehr, dies, um Schutz vor dem bei (allerdings sehr seltenen) explosionsartigen Ausbrüchen herunterfallenden Material zu bieten. Aus demselben Grund werden die Dächer niemals mit Ziegeln bedeckt, sondern bestehen aus massiven Balkenkonstruktionen, darüber Schilfrohr und ein leichter Mörtel aus vulkanischem Material. Diese Flachdächer sind ganz leicht geneigt, damit das kostbare Regenwasser in die Zisterne fließt. Früher wurden sie jeden Herbst sorgfältig abgedichtet und gekalkt. Bei längerer Trokkenheit griff man auf die beiden öffentlichen Reservoirs, gespeist durch die Dächer der Kirchen S. Vincenzo Ferreri und S. Bartolomeo. Seit 1954 bestehen außerdem zwei große Reservoirs, welche durch $\mathrm{Zi}$ sternenschiffe beliefert werden.

Ein wesentliches Element des Hauses auf Stromboli bildet die auf der Sonnenseite angeordnete Terrasse, teilweise von Rebspalier beschattet. Sie hat vielfältige Funktionen: hier werden Wäsche getrocknet, landwirtschaftliche Produkte verarbeitet (Feigen, Mandeln, Trauben, Tomaten, Kapern, Oliven), Gerätschaften repariert, außerdem spielt sich, typisch mediterran, ein bedeutender Teil des geselligen Lebens während

Dr. phil. II Peter A. Abt, Im Lett, 8634 Hombrechtikon 


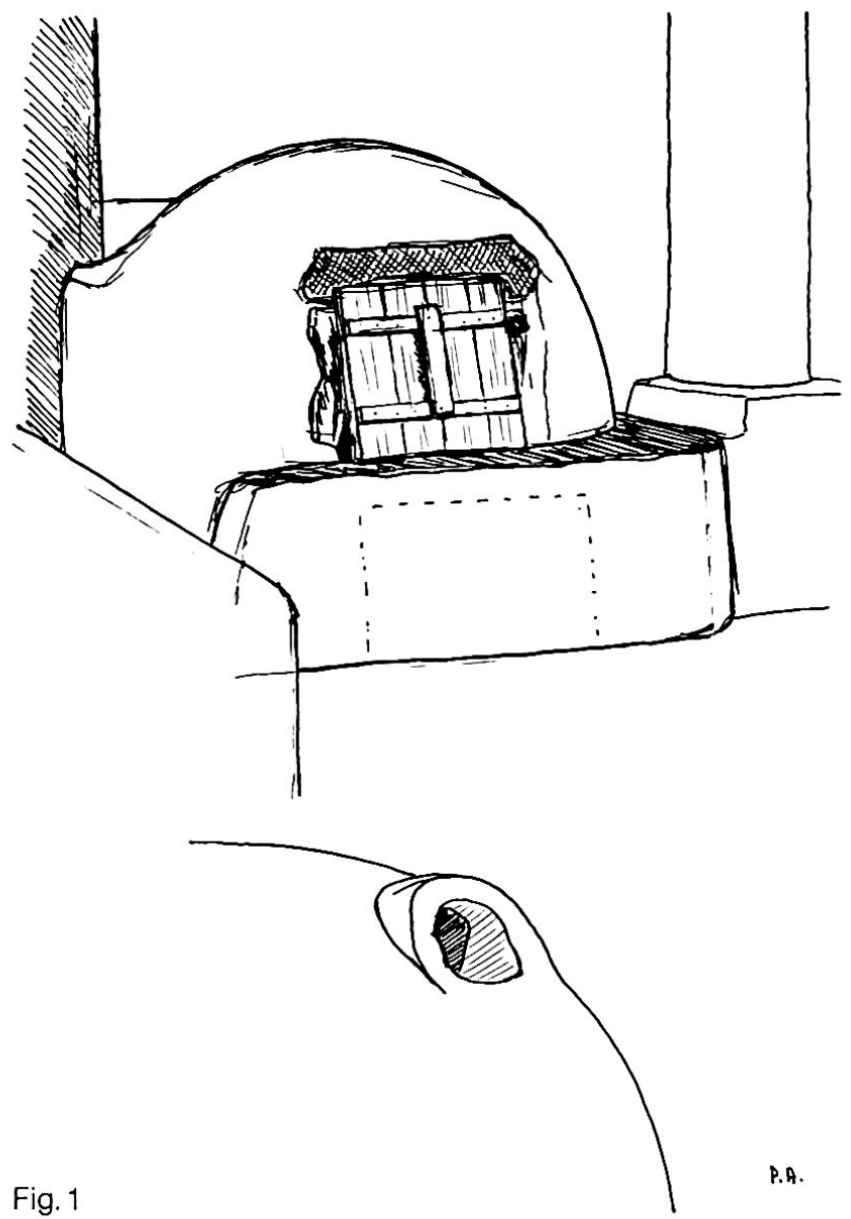

Fig. 1: Backofen im Ortsteil Ficogrande. Sorgfältig renoviert, seit Jahren außer Gebrauch. Gestrichelt: zugemauerter Raum für Brennholz. Auf der Rückseite: Rauchabzug, der beim Backen verschlossen wird.

Fig. 2: Haus am Strand von Ficogrande (neben Hotel Sirenetta). Zur Zeit unbewohnt, relativ schlechter Bauzustand. 2 Räume im Untergeschoß, 2 im Obergeschoß. 2 Backöfen. «Bauern-Fischer-Haus".

Fig. 3: Freistehender Backofen im Ortsteil Piscità, zerfallend. Diese ursprünglichen Typen wurden wohl wegen der Brandgefahr vom Haus entfernt erstellt (nach Famularo).

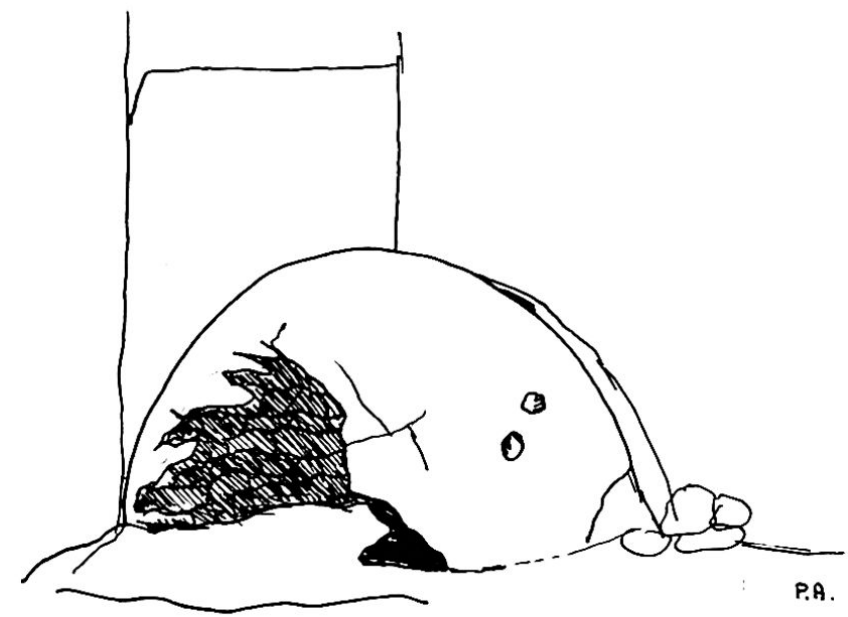

Fig. 3
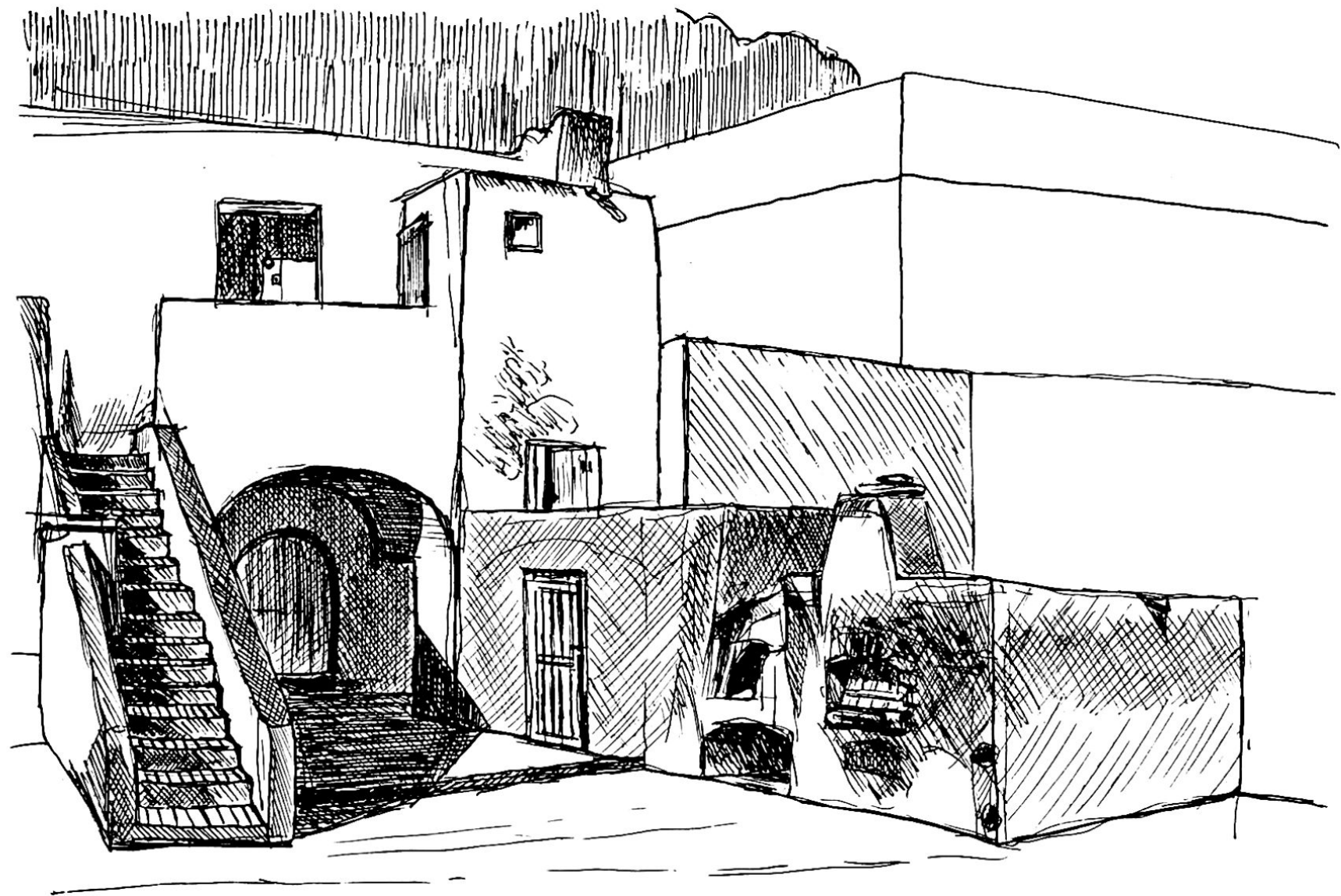

Fig. 2

P.A. 
der günstigen Jahreszeit hier ab. Im Winter dagegen versammelt man sich in der Küche, wo Herd und Backofen Wärme spenden. Oft sind die Backöfen für das Brot außerhalb angeordnet. Fig. 1 stellt einen kuppelförmigen, guterhaltenen Ofen dar, während in Fig. 2 eine quaderförmige Bauart festgehalten wurde, bei der noch ein kleinerer, kuppelförmiger Ofen für den normalen Küchenbedarf und zur Herstellung von Weihnachtsgebäck und anderen Spezialitäten, zwischengeschaltet ist. Eine urtümliche Form stellt Fig. 3 dar, dieser Ofen steht völlig frei im Garten. Anläßlich eines Besuches auf Stromboli im Sommer 1976 konnten wir noch da und dort Backöfen beobachten, die meisten allerdings in baufälligem Zustand. Schon seit längerer Zeit werden sie nicht mehr zum Backen verwendet, sondern dienen als Hühner- oder Kaninchenställe sowie zum Aufbewahren verschiedenster Gerätschaften.

Ein besonderes Kapitel widmet FAMULARo dem Wirtschaftsteil. Dieser hat gelegentlich bedeutende Ausmaße, nicht nur der vielfältigen Funktionen wegen, sonder aufgrund des (ehemals) vorherrschenden Weinbaues. Außerdem mußten in früheren Zeiten umfangreiche Vorräte an Nahrungsmitteln angelegt werden, da die winterlichen Stürme jegliche Verbindung mit dem Festland für viele Tage verunmöglichen konnten. Als wichtiges Stilelement wird die Treppe bezeichnet, welche entweder senkrecht zur Hauptfront (Fig. 2) oder, zweigeteilt, parallel dazu angeordnet ist. Die Stallungen für Kaninchen, Geflügel, Schafe und Ziegen werden irgendwo nahe beim Haus erstellt, während der Schweinestall, der Misthaufen und die Latrine möglichst weit entfernt sind.

In seinen Schlußbemerkungen weist FAMULARO nochmals auf die Tatsache hin, daß auch auf Stromboli im Zusammenhang mit dem aufstrebenden Tourismus der Prozeß der «deruralizzazione» immer stärker um sich greifen wird. Er hat diese Entwicklung selbst über rund ein halbes Jahrhundert verfolgen können. Tatsächlich ist ein Vergleich der Verhältnisse, wie sie in den Publikationen z. B. von WURM ${ }^{3)}$ oder CORAY ${ }^{4}$ ) geschildert werden, mit dem, was der Reisende heute vorfindet, frappant. Hoffen wir aber, FAMULARos Feststellung, daß die Häuser sich trotz vielfacher Erneuerungstendenzen nicht wesentlich verändert hätten, auch in Zukunft Gültigkeit behalte.

\section{Anmerkungen}

1) MIKUS, W.: Aspetti e problemi della geografica della popolazione nelle isole minori dell'Italia meridionale. Riv. Geogr. It. 76, 1969, H. 1, S. 15-54.

Ders: Vulkanische Inseln im Luftbild, in: Die Erde, Z. Ges. für Erdkunde zu Berlin, 100, 1969.

Ders.: Wirtschafts- und bevölkerungsgeographische Wandlungen auf den kleinen süditalienischen Inseln, in: Deutscher Geographentag Kiel, Tagungsbericht und wissenschaftliche Abhandlungen, Wiesbaden 1970. S. 440-462.

2) FAMULARO, $V .:$ La casa rurale nell'isola di Stromboli, Roma, 1971.

3) WURM, A.: Die äolischen Inseln, in: Deutsche Rundschau f. Geographie, XXXIV. Jahrgang, 1. H., 1911.

4) CORAY, H.: Bodenbearbeitung, ländliche Geräte, Ölbereitung, Weinbau und Fischerei auf den liparischen Inseln. Diss. Uni Zürich 1930. 